\title{
Arterial mechanical properties after replacement or reconstruction of the aortic root
}

\author{
Kai Mortensen ${ }^{1 *}$, Muhammet Aydin ${ }^{1 *}$, Alexander M. J. Bernhardt ${ }^{1}$, Verena Appenzeller ${ }^{1}$, \\ Peter N. Robinson ${ }^{2}$, Jürgen Berger ${ }^{1}$, Herman Reichenspurner ${ }^{1}$, Yskert von Kodolitsch ${ }^{1}$
}

${ }^{1}$ Centre of Cardiology and Cardiovascular Surgery at the University Hospital Eppendorf, Hamburg, Germany

${ }^{2}$ Institute for Medical and Human Genetics, Charité Universitätsmedizin, Berlin, Germany

Email: kodolitsch@uke.de

Received 4 October 2011; revised 14 December 2011; accepted 28 December 2011

\begin{abstract}
Background: Alteration of arterial mechanical properties has adverse effects on cardiovascular disease in Marfan syndrome. Design: We compared central pulse pressure, augmentation index adjusted to a heart rate of 75 beats per minute (AIx@HR75) and pulse wave velocity in 25 Marfan patients who had undergone an aortic valve-sparing reimplantation operation, in 15 Marfan patients after composite valve grafting operation, and in $\mathbf{4 0}$ age and sex-matched Marfan patients who had not undergone surgery. Results: Central pulse pressure, AIx@HR75 and pulse wave velocity were similar across all three patient groups. Exclusively AIx@HR75 was higher with conduit operations than in persons without any surgery $(P$ $=0.03$ ). Multivariate linear regression analysis documented association of AIx@HR75 with body height (P $<0.001)$ and with a history of aortic valve-sparing reimplantation operation $(P<\mathbf{0 . 0 0 1})$ or with a composite valve grafting operation $(P=0.006)$. Conclusions: Arterial mechanical properties are only mildly altered by surgery of the aortic root without difference between the reimplantation and conduit operation.
\end{abstract}

Keywords: Arterial Stiffness; Aortic Valve-Sparing Reimplantation Operation; Composite Valve Grafting Operation; Marfan Syndrome

\section{INTRODUCTION}

Alteration of arterial mechanical properties relate to cardiovascular events in healthy individuals, and in persons with cardiovascular diseases [1], including those with the Marfan syndrome [2]. The Marfan syndrome is an autosomal dominantly inherited disease of connective tissue which limits life expectancy mainly because of aortic rupture and dissection. Prophylactic replacement of the

"Both authors contributed equally to this work. aortic root with a composite valve grafting operation according to Bentall (CVG) has improved life expectancy of affected persons significantly. More recent surgical approaches have made it possible to preserve the native aortic valve [3]. We wanted to evaluate arterial mechanical properties in Marfan patients without any previous aortic root surgery and in patients after different types of aortic root surgery.

\section{AIM OF THE WORK}

We conducted this case-control study of the Marfan patients to compare arterial mechanical properties in persons who had undergone CVG, in persons after an aortic valve-sparing reimplantation operation according to David (AVR), and in an age and gender matched group of persons with the Marfan syndrome who had not undergone any aortic root operation.

\section{METHODS}

\subsection{Study Population}

We performed applanation tonometry in all patients with classical Marfan syndrome who underwent AVR or CVG $\geq 3$ months prior to tonometry. All patients were left on standard medication during applanation tonometry. All individuals had normal function of their aortic valve or of their aortic valve graft at the time of study and no patient exhibited more than grade I regurgitation or stenosis of any other heart valve. During the study period of 4 years we considered 90 consecutive post-surgical out-patients for inclusion in our study. Of these 90 persons, 25 individuals had aortic root surgery for acute type A aortic dissection, 20 persons underwent a total of 24 concomitant procedures including valve operations, atrial septal closure, and entire or partial replacement of aortic arch, and 6 had previously undergone other types of cardiovascular operations. The remaining 40 individuals were included in our study group that comprised 28 men and 12 women at a mean age of 42 years \pm 10 years (range 
20 years - 64 years; Table 1). Our control patients all had classical Marfan syndrome, they had applanation tonometry during the same time interval as in our study patients, and they had not undergone any cardiovascular surgery or intervention [2]. We matched each study patient with one of these individuals for age, sex and body mass index and thus identified 28 men and 12 women at a mean age of 43 years \pm 12 years (range 19 years - 71 years) as our control patients (Table 2).

\subsection{Study Variables}

We originally established Marfan syndrome with uniform use of the classical Ghent criteria, but we confirmed compliance with the recently revised Ghent nosology in all 80 individuals $[4,5]$. We performed the AVR operation with reimplantation of the native aortic valve into a straight tube graft as originally described by David and Feindel [6] and as classified David-I according to Miller [7] in all 25 patients. Similarly, we used the same variant of the CVG procedure for aortic root replacement in all 15 individuals where we reimplanted the coronary arteries with the button technique [8] (Table 1).

We assessed age at the time of applanation tonometry, body weight, body height, and body mass index in all individuals. We considered active smoking with any inhalative intake of nicotine within 7 days of the study and we assessed fasting blood glucose levels and fasting lipid levels $\leq 24$ hours of applanation tonometry. We performed standard M-mode, 2-dimensional and color coded transthoracic echocardiography according to our published protocols [9]. We measured maximum aortic diameters at the level of the aortic sinuses on transthoracic echocardiography, with calculation of aortic root ratios as described by Roman et al. [10]. We measured the diameter of the ascending aorta and the descending aorta on

Table 1. Operative data in 40 consecutive Marfan patients with elective aortic root replacement.

\begin{tabular}{lccc}
\hline Variables & AVR & CVG & $P^{\mathrm{a}}$ \\
\hline Procedures, number & 25 & $15^{\mathrm{b}}$ & - \\
Age at aortic root surgery; years & $39 \pm 10$ & $38 \pm 14$ & 0.89 \\
Extracorporeal circulation (min) & $161 \pm 34$ & $122 \pm 46$ & $<0.001$ \\
Aortic cross-clamping (min) & $122 \pm 33$ & $77 \pm 27$ & $<0.001$ \\
Biological AVP & - & $5(33 \%)$ & - \\
Monodisc AVP & - & $2(13 \%)$ & - \\
Bileaflet AVP & - & $8(53 \%)$ & - \\
Diameter of AVP (mm) & - & $27 \pm 2$ & - \\
Diameter of aortic tube graft (mm) & $29 \pm 2$ & $30 \pm 3$ & 0.37 \\
\hline
\end{tabular}

AVR indicates aortic valve-sparing reimplantation operation, and CVG, composite valve grafting operation, AVP, aortic valve prosthesis. ${ }^{a}$ Mann-Whitney test for continuous data and the Fisher's exact test for nominal and categorycal data. ${ }^{\mathrm{b}}$ Two patients had concomitant coronary artery bypass grafting.
Table 2. Demographic and clinical characteristics in 80 Marfan patients.

\begin{tabular}{|c|c|c|c|c|}
\hline \multirow[b]{2}{*}{ Variables } & \multicolumn{4}{|c|}{ Aortic root surgery } \\
\hline & $\begin{array}{c}\text { No } \\
\text { surgery } \\
(N=40)\end{array}$ & $\begin{array}{c}A V R \\
(N=25)\end{array}$ & $\begin{array}{c}C V G \\
(N=15)\end{array}$ & $\begin{array}{c}\text { Global } \\
\text { test }{ }^{a}\end{array}$ \\
\hline Age; years & $43 \pm 12$ & $41 \pm 9$ & $43 \pm 11$ & 0.63 \\
\hline Male gender & $28(70 \%)$ & $18(72 \%)$ & $10(67 \%)$ & 0.94 \\
\hline Body weight $(\mathrm{kg})$ & $78 \pm 12$ & $88 \pm 20$ & $79 \pm 20$ & $0.04^{\mathrm{b}}$ \\
\hline Body height $(\mathrm{cm})$ & $1.85 \pm 0.11$ & $1.93 \pm 0.08$ & $1.86 \pm 0.1$ & $0.02^{\mathrm{c}}$ \\
\hline BMI $\left(\mathrm{kg} / \mathrm{m}^{2}\right)$ & $22.8 \pm 3.2$ & $23.7 \pm 5.1$ & $22.7 \pm 2.2$ & 0.6 \\
\hline Current smoking & $3(8 \%)$ & $5(20 \%)$ & $2(13 \%)$ & 0.34 \\
\hline Blood glucose (mg/dl) & $102 \pm 59$ & $94 \pm 18$ & $101 \pm 31$ & 0.78 \\
\hline $\mathrm{TC}(\mathrm{mg} / \mathrm{dl})$ & $184 \pm 52$ & $182 \pm 33$ & $209 \pm 36$ & 0.12 \\
\hline $\operatorname{HDL}(\mathrm{mg} / \mathrm{dl})$ & $57 \pm 15$ & $54 \pm 13$ & $55 \pm 14$ & 0.85 \\
\hline $\operatorname{LDL}(\mathrm{mg} / \mathrm{dl})$ & $111 \pm 41$ & $104 \pm 26$ & $126 \pm 38$ & 0.21 \\
\hline Aortic root ratio & $1.14 \pm 0.2$ & $0.88 \pm 0.24$ & $0.94 \pm 0.08$ & $<0.001^{\mathrm{d}}$ \\
\hline $\mathrm{AAD}(\mathrm{cm})$ & $3 \pm 0.5$ & $2.9 \pm 0.3$ & $3 \pm 0.4$ & 0.27 \\
\hline $\mathrm{DAD}(\mathrm{cm})$ & $2.3 \pm 0.5$ & $2.5 \pm 0.6$ & $2.3 \pm 0.3$ & 0.26 \\
\hline LVESD/BSA $\left(\mathrm{mm} / \mathrm{m}^{2}\right)$ & $21 \pm 6.3$ & $19.6 \pm 5.2$ & $20.3 \pm 3.6$ & 0.66 \\
\hline $\operatorname{LVEDD} / \mathrm{BSA}\left(\mathrm{mm} / \mathrm{m}^{2}\right)$ & $27 \pm 5.1$ & $26.2 \pm 6.2$ & $27.5 \pm 5.8$ & 0.77 \\
\hline $\mathrm{LAD} / \mathrm{BSA}\left(\mathrm{mm} / \mathrm{m}^{2}\right)$ & $20.2 \pm 6.3$ & $18.6 \pm 3.7$ & $18 \pm 4.1$ & 0.32 \\
\hline LVEF (\%) & $54 \pm 11$ & $55 \pm 11$ & $57 \pm 8$ & 0.67 \\
\hline NT-proBNP (pg/ml) & $145 \pm 156$ & $245 \pm 214$ & $286 \pm 214$ & 0.1 \\
\hline Beta-blockers & $17(43 \%)$ & $15(60 \%)$ & $8(53 \%)$ & 0.37 \\
\hline Angiotensin-antagonists & $9(23 \%)$ & $12(48 \%)$ & $5(33 \%)$ & 0.1 \\
\hline
\end{tabular}

Data are presented as the mean value $\pm \mathrm{SD}$ or number (percent) of patients. No surgery indicate Marfan patients without any aortic surgery; AAD, ascending aorta diameter; BMI, body mass index; DAD, descending aorta diameter; HDL, high-density lipoprotein cholesterol; LAD/BSA, indexed left atrial diameter; LDL, low-density lipoprotein cholesterol; LVEF, left ventricular ejection fraction; LVEDD/BSA, indexed end-diastolic left ventricular diameter; LVESD/BSA, indexed end-systolic left ventricular diameter; NT-proBNP, N-terminal pro-brain natriuretic peptide; TC, total cholesterol. All other abbreviations are as indicated in Table 1. ${ }^{\mathrm{a}}$ Freeman-Halton test for categorical variables and analysis of variance for continuous variables. Bonferroni test: ${ }^{\mathrm{b}}$ No surgery vs Valve-sparing $P=0.04$, and vs Composite grafting $P=1$, Valve-sparing vs Composite grafting $P=0.23$, ${ }^{\mathrm{C}}$ No surgery vs Valve-sparing $P=0.02$, and vs Composite grafting $P=1$, Valve-sparing vs Composite grafting $P=0.2 .{ }^{\mathrm{d}}$ No surgery vs Valve-sparing $P<0.001$, and vs Composite grafting $P=0.007$, Valve-sparing vs Composite grafting $P=1$. 
magnetic resonance angiography at established levels [11]. We assessed left ventricular ejection fraction, endsystolic left ventricular diameter, end-diastolic left ventricular diameter, and left atrial diameter on two-dimensional echocardiography according to current guidelines [12], and we adjusted for differences in body size by dividing left ventricular and atrial diameters by body surface area as calculated by the formula of Du Bois [13]. We documented medication with beta-blockers, angiotensin converting enzyme inhibitors, or angiotensin-receptor blockers with chronic intake of standard dosages (Table 2).

\subsection{Tonometry}

We performed applanation tonometry according to international guidelines [14] using our previously described protocols [2]. All investigators were blinded to clinical data, diagnoses, or therapy of all individuals. We accepted measurements as valid only with standard deviation of beat-to-beat data $<10 \%$ of its mean and when complying with the internal SphygmoCor ${ }^{\circledR}$ quality control criteria [2]. We obtained radial artery waveforms from the wrist of the dominant arm using a high-fidelity micromanometer (SPC-301; Millar Instruments, Houston, TX) [2]. A validated transfer function (SphygmoCor ${ }^{\circledR}$; AtCor Medical, Sydney, Australia) generated the corresponding central waveforms, and the integral SphygmoCor ${ }^{\circledR}$ software determined central blood pressures, augmentation index, the time to the peak/shoulder of the first (T1) and second pressure wave components (T2) during systole, the time to return of the reflected pressure wave (Tr), ejection duration, central pulse pressure, and heart rates as described previously [2]. We assessed the augmentation index adjusted to a heart rate of 75 beats per minute (AIx@HR75). We obtained the mean arterial pressure, and the subendocardial viability ratio (Buckberg's ratio) from integrals of the central waveform. We measured the blood pressure level oscillometrically at the dominant upper arm (Omron HEM 750; Omron Healthcare, Kyoto, Japan; Table 2). We measured the carotid-femoral pulse wave velocity as described previously in all individuals [15].

\section{STATISTICAL ANALYSIS}

We expressed quantitative data as means ( \pm standard deviation), and qualitative data as numbers and proportions (percentage). We compared qualitative and categorical data by the generalized Fisher's exact test (FreemanHalton test) and quantitative data by the Mann-Whitney test or by analysis of variance (Tables 1-3). We performed stepwise multivariable regression analysis $(\mathrm{Pin}=0.05$, Pout $=0.10)$ to evaluate the independent contribution of each demographic and clinical variable that was associated with AIx@HR75 at a level of significance set at $P \leq$
0.1 (2-sided). For all associations, we provided the linear regression coefficients $(\beta)$ and their corresponding 95\% confidence intervals (95\% CIs) for continuous variables or an odds ratio and their corresponding 95\% CI for dichotomous variables. We used SPSS software (SPSS for Windows, Release 17.0, SPSS Inc. 1993 to 2007, Chicago, Illinois) for all statistical analyses.

Table 3. Hemodynamic findings in 80 Marfan patients.

\begin{tabular}{|c|c|c|c|c|}
\hline \multirow[b]{2}{*}{ Variables } & \multicolumn{4}{|c|}{ Aortic root surgery } \\
\hline & $\begin{array}{c}\text { No } \\
\text { surgery } \\
(N=40)\end{array}$ & $\begin{array}{c}A V R \\
(N=25)\end{array}$ & $\begin{array}{c}C V G \\
(N=15)\end{array}$ & $\begin{array}{c}\text { Global } \\
\text { test }^{a}\end{array}$ \\
\hline Heart rate (beats/min) & $63 \pm 11$ & $66 \pm 8$ & $66 \pm 11$ & 0.39 \\
\hline PSBP $(\mathrm{mmHg})$ & $127 \pm 14$ & $127 \pm 12$ & $126 \pm 14$ & 0.99 \\
\hline PDBP (mmHg) & $70 \pm 9$ & $69 \pm 9$ & $70 \pm 12$ & 0.76 \\
\hline PMBP $(\mathrm{mmHg})$ & $89 \pm 11$ & $90 \pm 14$ & $90 \pm 11$ & 0.91 \\
\hline $\mathrm{CSBP}(\mathrm{mmHg})$ & $113 \pm 13$ & $115 \pm 18$ & $117 \pm 14$ & 0.66 \\
\hline $\mathrm{CDBP}(\mathrm{mmHg})$ & $71 \pm 9$ & $69 \pm 9$ & $71 \pm 12$ & 0.78 \\
\hline CMBP (mmHg) & $87 \pm 17$ & $90 \pm 14$ & $90 \pm 11$ & 0.64 \\
\hline $\mathrm{T} 1$ (ms) & $113 \pm 19$ & $105 \pm 15$ & $114 \pm 26$ & 0.25 \\
\hline $\mathrm{T} 2(\mathrm{~ms})$ & $223 \pm 27$ & $218 \pm 23$ & $225 \pm 19$ & 0.61 \\
\hline $\operatorname{Tr}(\mathrm{ms})$ & $148 \pm 21$ & $141 \pm 25$ & $147 \pm 30$ & 0.51 \\
\hline SEVR (\%) & $159 \pm 29$ & $142 \pm 24$ & $153 \pm 35$ & 0.08 \\
\hline $\mathrm{ED}(\mathrm{ms})$ & $331 \pm 29$ & $330 \pm 33$ & $323 \pm 30$ & 0.64 \\
\hline PPP (mmHg) & $57 \pm 8$ & $58 \pm 8$ & $57 \pm 14$ & 0.81 \\
\hline $\mathrm{CPP}(\mathrm{mmHg})$ & $42 \pm 8$ & $43 \pm 8$ & $46 \pm 14$ & 0.33 \\
\hline AIx@HR75 (\%) & $14 \pm 14.8$ & $21.5 \pm 13.8$ & $25.4 \pm 12.1$ & $0.02^{b}$ \\
\hline $\mathrm{PWV}(\mathrm{m} / \mathrm{s})$ & $7.28 \pm 2.3$ & $6.53 \pm 1.92$ & $6.3 \pm 2.35$ & 0.24 \\
\hline
\end{tabular}

Data are presented as the mean value \pm SD or number (percent) of patients. AIx@HR7, augmentation index adjusted to a heart rate of 75 beats per minute; CDBP, central diastolic blood pressure; CMBP, central mean blood pressure; CSBP, central systolic blood pressure; CPP, central pulse pressure; $\mathrm{ED}$, ejection duration; PDBP, peripheral diastolic blood pressure; PMBP, peripheral mean blood pressure; PPP, peripheral pulse pressure; PSBP, peripheral systolic blood pressure; PWV, carotid-femoral pulse wave velocity; SEVR, subendocardial viability ratio; $\mathrm{T} 1$, aortic $\mathrm{T} 1$; T2, aortic $\mathrm{T} 2$; and $\mathrm{Tr}$, time to return of reflected pulse wave. All other abbreviations are as indicated in Table1. ${ }^{a}$ Mann-Whitney test for continuous data and the Fisher's exact test for nominal and categorical data. Bonferroni test: ${ }^{b}$ No surgery vs Valve-sparing $P=0.18$, and vs Composite grafting $P=0.03$, Valve-sparing vs Composite grafting $P=1$. 


\section{RESULTS}

The operative data of our 25 individuals with an AVR and of the 15 individuals with a CVG are shown in Table 1. The body weight $(P=0.04)$ and the body height $(P$ $=0.02$ ) was higher in individuals who had undergone AVR than in persons without surgery, and the aortic root ratios were smaller after AVR $(P<0.001)$ or after CVG $(P=0.007)$ than in individuals without aortic root surgery (Table 2). Hemodynamic findings at applanation tonometry were similar in all Marfan patients irrespective of presence or type of aortic root surgery. AIx@HR75 was slightly higher after CVG than in individuals after AVR but differences were significant only between persons after CVR and persons without any aortic surgery (Table 3; Figures 1-2). Multivariate linear regression analysis of body weight, body height, aortic root ration, N-terminal pro-brain natriuretic peptide, and presence of an aortic root operation showed an association of AIx@HR75 only with body height (regression coefficient -84.679 ; 95\% confidence interval (CI)-116.6-52.7; $P<0.001$ ), with AVR (regression coefficient 15.2; 95\% CI 7.141-23.252; $P<0.001$ ), and with CVG (regression coefficient 12.04; 95\% CI-3.7-20. 4; $P=0.006$ ).

\section{DISCUSSION}

Assessment of arterial mechanical properties is increaseingly recognized as a useful aid to identify an increased cardiovascular risk in the Marfan syndrome and related disorders $[2,15]$. Central pulse pressure is a direct measure of arterial stiffness whereas central pulse pressure and Aix@HR75 is considered an important indirect measure of arterial mechanical properties [1]. Although the pathophysiology of increased AIx@HR75 is not yet fully understood [16], it is well-known, that in younger patients with premature aortic wall degeneration AIx@HR75 increases with presence of still normal pulse wave velocities $[2,15,17]$. In this study, pulse wave velocities and AIx@HR75 were similar in all Marfan patients and AIx@ HR75 was which elevated only after CVG as compared to persons without any aortic surgery.

The effect of aortic root replacement surgery on arterial mechanical properties has not been investigated to date. However, a previous study of patients after tube graft implantation for abdominal aortic aneurysms showed an increase of AIx@HR75 and pulse wave velocity [18]. Our study exclusively demonstrated an increase of AIx@HR75 after CVG. Most likely pulse wave velocity was not increased because we measured carotid-femoral pulse wave velocities which did not include the proximal aortic vessel segment. Moreover, the difference of AIx@ HR75 in postsurgical patients and in patients without previous aortic root surgery was only small, probably because non-surgical Marfan patients have a diseased aortic root which already exhibits impaired arterial tissue function [2].

In contrast to CVG reconstructive surgery of the aortic root preserves the native aortic valve. It has been suggested that with the native valve tissue left in place the tissue elasticity and function of the aortic root might be preserved [19]. However, AVR did not yield better Aix@ HR75 and we did not find any differences of arterial stiffness parameters with the use of an AVR operation as compared to the complete replacement of the aortic root. This finding may in be explained by our use of the David-I variant of AVR, where the semilunar aortic valves are

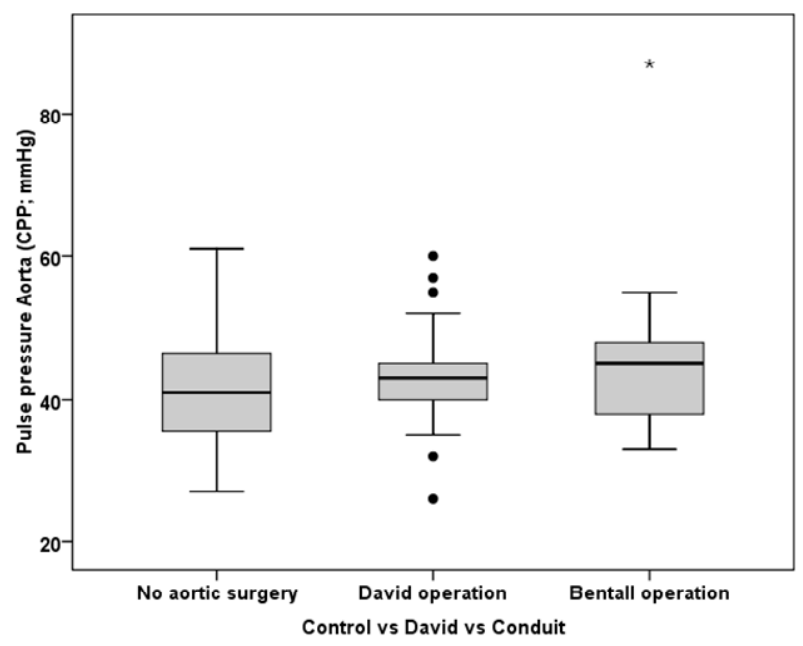

Figure 1. The box-and-whiskers plot of central pulse pressure according to presence of aortic root surgery. The boxes indicate the upper and lower quartile and the median (horizontal lines), and the whiskers identifying the minimum and maximum values. Individuals with David operation or a composite valve grafting operation (Bentall operation) exhibited similar central pulse pressure as individuals without any aortic root surgery.

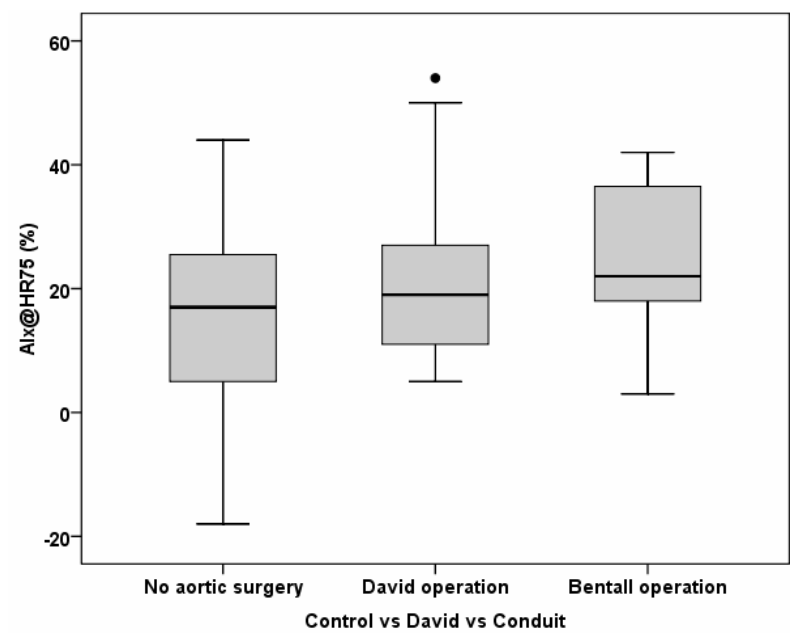

Figure 2. The box-and-whiskers plot of AIx@HR75 according to presence of aortic root surgery. AIx@HR75 was only somewhat higher in individuals after a David operation than in individuals without aortic root surgery $(P=0.03$; lower panel). 
trapped by being implanted entirely into the aortic tube graft, and which may thus prevent them from contributing to arterial elasticity. However, differences of nonsurgical and surgical Marfan patients were only minor. As it is known from previous studies, the native tissue of the aortic root is already associated with reduced arterial mechanical property function $[2,15]$. Our current data suggest that both replacement and reconstruction of the aortic root do not contribute significantly to a further reduction of Windkessel function in Marfan patients.

The left ventricular ejection fraction and indexed endsystolic and end-diastolic left ventricular diameters did not differ between surgical patients and individuals without aortic surgery. Moreover, we did not observe differrences of left ventricular function or geometry between patients with both types of operation. Conversely, N-terminal pro-brain natriuretic peptide levels as a serologic marker of diastolic left ventricular dysfunction were slightly higher after CVG than after AVR or in patients without aortic root operation. In various diseases left ventricular dysfunction is associated with increased arterial stiffness, and we also found that increased $\mathrm{N}$-terminal probrain natriuretic peptide levels are related to higher AIx@HR75 on univariate linear regression analysis (regression coefficient $0.024 ; 95 \% \mathrm{CI} 0.005-0.42 ; P=0.01$ ). However, multivariate regression analysis showed a relationship of AIx@HR75 with presence of an aortic root operation but not with $\mathrm{N}$-terminal pro-brain natriuretic peptide levels.

\section{Study Limits}

Some study limits should be addressed. First, our patient numbers were low and small differences of hemodynamic measurements may become significant in studies with larger patient numbers. Moreover, there were only 15 patients with different types of aortic valves in the CVG group, and differences of these devices may have accounted for different measurements. However, we compared patients with isolated AVR or with isolated CVG carried out for identical indications, which were isolated aneurysms of the aortic root without both aortic dissection and the need for extending surgery into the aortic arch. Moreover, all patients had similar age at the time of study. Most importantly, we exclusively included patients with replacement of the proximal aorta and the aortic tube grafts were tailored to a length of $1.5 \mathrm{~cm}$ beyond the origin of the brachiocephalic trunk in all patients with AVR and CVG. Thus, our findings were unlikely to be severely confounded by intervening variables such as different lengths of tube grafts or concomitant surgical procedures.

\section{CONCLUSION}

Our preliminary data suggest that arterial mechanical pro- perties are slightly better in Marfan patients without any surgery but they do not exhibit differences between patients after aortic valve-sparing operations and after composite valve grafting operations. For further corroboration of these findings future studies may assess mechanical arterial properties in larger patient groups and in other variants of AVR including those with reconstructtion of the sinuses.

\section{REFERENCES}

[1] Laurent, S., Cockcroft, J., Van Bortel, L., et al. (2006) Expert consensus document on arterial stiffness: Methodological issues and clinical applications. European Heart Journal, 27, 2588-2605. doi:10.1093/eurhearti/ehl254

[2] Mortensen, K., Aydin, M.A., Rybczynski, M., et al. (2009) Augmentation index relates to progression of aortic disease in adults with Marfan syndrome. American Journal of Hypertension, 22, 971-979. doi:10.1038/ajh.2009.115

[3] Karck, M., Kallenbach, K., Hagl, C., et al. (2004) Aortic root surgery in Marfan syndrome: Comparison of aortic valve-sparing reimplantation versus composite grafting. The Journal of Thoracic and Cardiovascular Surgery, 127, 391-398. doi:10.1016/j.jtcvs.2003.07.049

[4] Loeys, B.L., Dietz, H.C., Braverman, A.C., et al. (2010) The revised Ghent nosology for the Marfan syndrome. Journal of Medical Genetics, 47, 476-485. doi:10.1136/jmg.2009.072785

[5] Sheikhzadeh, S., Kade, C., Keyser, B., et al. (2011) Analysis of phenotype and genotype information for the diagnosis of Marfan syndrome. Clinical Genetics, in press. doi:10.1111/j.1399-0004.2011.01771.x

[6] David, T.E. and Feindel, C.M. (1992) An aortic valvesparing operation for patients with aortic incompetence and aneurysm of the ascending aorta. The Journal of Thoracic and Cardiovascular Surgery, 103, 617-622.

[7] Miller, D.C. (2003) Valve sparing aortic root replacement in patients with the Marfan syndrome. The Journal of Thoracic and Cardiovascular Surgery, 125, 773-778. doi:10.1067/mtc.2003.162

[8] Bernhardt, A.M., Treede, H., Rybczynski, M., et al. (2011) Comparison of aortic root replacement in patients with Marfan syndrome. European Journal of Cardiothoracic Surgery, 40, 1052-1057. doi:10.1016/j.ejcts.2011.02.018

[9] Rybczynski, M., Koschyk, D.H., Aydin, M.A., et al. (2007) Tissue Doppler imaging identifies myocardial dysfunction in adults with Marfan syndrome. Clinical Cardiology, 30, 19-24. doi:10.1002/clc.3

[10] Roman, M.J., Devereux, R.B., Kramer-Fox, R. and O'Loughlin, J. (1989) Two-dimensional echocardiographic aortic root dimensions in normal children and adults. American Journal of Cardiology, 64, 507-512. doi:10.1016/0002-9149(89)90430-X

[11] Groenink, M., de Roos, A., Mulder, B.J., Spaan, J.A. and van der Wall, E.E. (1998) Changes in aortic distensibility and pulse wave velocity assessed with magnetic resonance imaging following beta-blocker therapy in the Mar- 
fan syndrome. American Journal of Cardiology, 82, 203208. doi:10.1016/S0002-9149(98)00315-4

[12] Schiller, N.B., Shah, P.M., Crawford, M., et al. (1989) Recommendations for quantitation of the left ventricle by two-dimensional echocardiography. American Society of Echocardiography Committee on Standards, Subcommittee on Quantitation of Two-Dimensional Echocardiograms. Journal of the American Society Echocardiography, 2, 358-367.

[13] Du Bois, D. and Du Bois, E.F. (1989) A formula to estimate the approximate surface area if height and weight be known. Nutrition, 5, 303-311.

[14] Van Bortel, L.M., Duprez, D., Starmans-Kool, M.J., et al. (2002) Clinical applications of arterial stiffness, Task Force III: Recommendations for user procedures. American Journal of Hypertension, 15, 445-452. doi:10.1016/S0895-7061(01)02326-3

[15] Mortensen, K., Baulmann, J., Rybczynski, M., et al. (2010) Augmentation index and the evolution of aortic disease in marfan-like syndromes. American Journal of Hypertension, 23, 716-724. doi:10.1038/ajh.2010.78

[16] Payne, R.A. (2009) Augmenting the assessment of Marfan syndrome? American Journal of Hypertension, 22, 951. doi:10.1038/ajh.2009.129

[17] Payne, R.A., Hilling-Smith, R.C., Webb, D.J., Maxwell, S.R. and Denvir, M.A. (2007) Augmentation index assessed by applanation tonometry is elevated in Marfan Syndrome. Journal of Cardiothoracic Surgery, 2, 43-49. doi:10.1186/1749-8090-2-43

[18] Lantelme, P., Dzudie, A., Milon, H., et al. (2009) Effect of abdominal aortic grafts on aortic stiffness and central hemodynamics. Journal of Hypertension, 27, 1268-1276. doi:10.1097/HJH.0b013e3283299b22

[19] Yacoub, M.H. and Cohn, L.H. (2004) Novel approaches to cardiac valve repair: From structure to function: Part I. Circulation, 109, 942-950. doi:10.1161/01.CIR.0000115633.19829.5E 